\title{
III-nitride nanowire LEDs and diode lasers: monolithic light sources on (001) Si emitting in the 600-1300nm range
}

P. Bhattacharya, A. Hazari, S. Jahangir

P. Bhattacharya, A. Hazari, S. Jahangir, "III-nitride nanowire LEDs and diode lasers: monolithic light sources on (001) Si emitting in the 600-1300nm range," Proc. SPIE 10553, Novel In-Plane Semiconductor Lasers XVII, 1055302 (19 February 2018); doi: 10.1117/12.2302548

SPIE. Event: SPIE OPTO, 2018, San Francisco, California, United States 
Plenary Paper

\title{
III-Nitride Nanowire LEDs and Diode Lasers: Monolithic Light Sources on (001) Si Emitting in the 600-1300nm Range
}

\author{
P. Bhattacharya, A. Hazari, and S. Jahangir \\ Department of Electrical Engineering and Computer Science \\ University of Michigan, Ann Arbor, MI 48109-2122, USA
}

\begin{abstract}
GaN-based nanowire heterostructure arrays epitaxially grown on (001)Si substrates have unique properties and present the potential to realize useful devices. The active light-emitting region in the nanowire heterostructures are usually InGaN disks, whose composition can be varied to tune the emission wavelength. We have demonstrated light emitting diodes and edgeemitting diode lasers with power outputs $\sim 10 \mathrm{~mW}$ with emission in the $600-1300 \mathrm{~nm}$ wavelength range. These light sources are therefore useful for a variety of applications, including silicon photonics. Molecular beam epitaxial growth of the nanowire heterostructure arrays on (001)Si substrates and the characteristics of $1.3 \mu \mathrm{m}$ nanowire array edge emitting lasers, guided wave photodiodes and a monolithic photonic integrated circuit designed for $1.3 \mu \mathrm{m}$ operation are described.
\end{abstract}

Keywords: III-nitrides, InGaN/GaN and InGaN/InGaN dot-in-nanowires, Lasers and detectors on (001)Si, monolithic photonic integrated circuit, molecular beam epitaxy

\section{INTRODUCTION}

III-nitride based nanowires and quantum-confined heterostructures have emerged as a promising nanostructured technology for the development of visible light emitting diodes (LEDs) and lasers [1]-[3]. Ga(In)N nanowires grown on (001) silicon by molecular beam epitaxy (MBE) are relatively free of extended defects compared to planar GaN grown on foreign substrates [4]-[9]. The nanowire geometry prevents the propagation of threading dislocations into the active region of the heterostructure because the free surface at the nanowire sidewalls allows for the elastic relaxation of the strain [10]. Therefore, threading dislocations generated at nanowire/silicon interface are expected to bend toward the sidewalls near the bottom of the nanowires (NWs) [11]. Auger coefficients measured in InGaN/GaN disks-in-nanowires are $\sim 2-3$ orders of magnitude smaller than those measured in heteroepitaxial bulk materials [12]. This is important in the context of LED and laser efficiency at high injection currents. Achieving emission at longer wavelengths (green and beyond) with planar InGaN quantum wells (QWs) with high radiative efficiency is difficult. This is due to the strong polarization field and associated quantum-confined Stark effect (QCSE) [13], the presence of compositional inhomogeneities in the ternary alloys with high In composition [14], [15] and an increasing rate of non-radiative recombination due to the lack of confinement of carriers in the in-plane direction in the wells. It has been reported that the internal quantum efficiency of planar quantum wells decreases with an increase of emission wavelength at a rate of $\sim 0.6-0.7 \%$ per nanometer [16]. In contrast, $\mathrm{Ga}(\mathrm{In}) \mathrm{N}$ nanowires and $\mathrm{InGaN} / \mathrm{GaN}$ disks-in-nanowires (DNW) have reduced strain-induced polarization field even with high In compositions. This is due to the radial strain relaxation during their epitaxy resulting from their large surface-to-volume ratio. As a

Novel In-Plane Semiconductor Lasers XVII, edited by Alexey A. Belyanin, Peter M. Smowton, Proc. of SPIE Vol. 10553, 1055302 - (C) 2018 SPIE · CCC code: 0277-786X/18/\$18 · doi: 10.1117/12.2302548 
consequence, electron-hole wavefunction overlap is better and radiative lifetimes are smaller in the disks [17]. Accessibility to the longer wavelengths, such as $600 \mathrm{~nm}$ and beyond, could then very well emerge as the most useful attribute of nanowire heterostructures in the context of solid state lighting.

Current white light sources in solid state lighting require the use of rare-earth doped phosphors, e.g. for the conversion of blue light to the yellow-red spectral range [18]-[22]. Such conversion is inevitably accompanied by a variety of losses and is limited by long-term reliability [16], [23]-[25]. Combining multiple colors with the same material system, either as direct emitters (R-G-Y-B) or as a combination of internal converter and emitter, is an attractive alternative. InGaN ternary alloys with different In compositions cover the entire visible range in bandgap from blue to red emission. Therefore, they could in principle, provide a white light source with any desired color coordinates and color temperature [2], [3], [26], [27]. Increasing the In composition in the InGaN disks in GaN nanowires further can result in near-infrared (near-IR) emission which is important for silicon photonics. However, before these important applications could be realized, the epitaxy and optical properties of the nanowire heterostructures had to be characterized and optimized. While it has been reported that the surface recombination velocity of GaN nanowires is smaller than that of GaAs by 2 orders of magnitude [28], the injected carriers would encounter a large area of nanowire surface with a detrimental effect on the radiative efficiency [29], [30]. Due to their large surface-to-volume ratio and the presence of surface states on the nanowires, there can be substantial nonradiative recombination of carriers through these states-leading to reduced radiative efficiency. Hence, surface passivation of nanowires is likely to play a significant role in the improvement of the radiative efficiency of the $\mathrm{Ga}(\mathrm{In}) \mathrm{N}$ disks and nanowires.

The epitaxy and properties of dot-in-nanowire heterostructure array visible LEDs and lasers, and nanowire converter white LEDs have been extensively reported by us in previous publications [31]-[33]. Here, we will focus on describing the characteristics of near-infrared 1.2 and $1.3 \mu \mathrm{m}$ nanowire diode lasers and the monolithic integration of the lasers with nanowire detectors and passive waveguides to form a photonic integrated circuit.

\section{1.2 $2 \mathrm{M}$ DISK-IN-NANOWIRE DIODE LASERS}

The laser heterostructure consists of $\operatorname{In}_{0.85} \mathrm{Ga}_{0.15} \mathrm{~N}$ disks in the active region as shown in Fig. 1. The monolithic InGaN/InGaN disks-innanowire array $\mathrm{p}-\mathrm{i}-\mathrm{n}$ heterostructures were grown on n-type (001)Si by plasma-assisted molecular beam epitaxy (PA-MBE). The growth was carried out in a nitrogen rich environment with a fixed nitrogen plasma flowrate of $1 \mathrm{sccm}$. First, $260 \mathrm{~nm}$ of Sidoped n-type GaN nanowire was grown. This was followed by a n-type $150 \mathrm{~nm}$ graded cladding layer from $\mathrm{GaN}$ to $\mathrm{In}_{0.4} \mathrm{Ga}_{0.6} \mathrm{~N}$ in 10 equal steps. The steps were equal both in terms of composition and thickness. Then eight pairs of undoped $\operatorname{In}_{0.85} \mathrm{Ga}_{0.15} \mathrm{~N}$ $(3 \mathrm{~nm})$ disks/ $\mathrm{In}_{0.4} \mathrm{Ga}_{0.6} \mathrm{~N}(12 \mathrm{~nm})$ barrier were grown

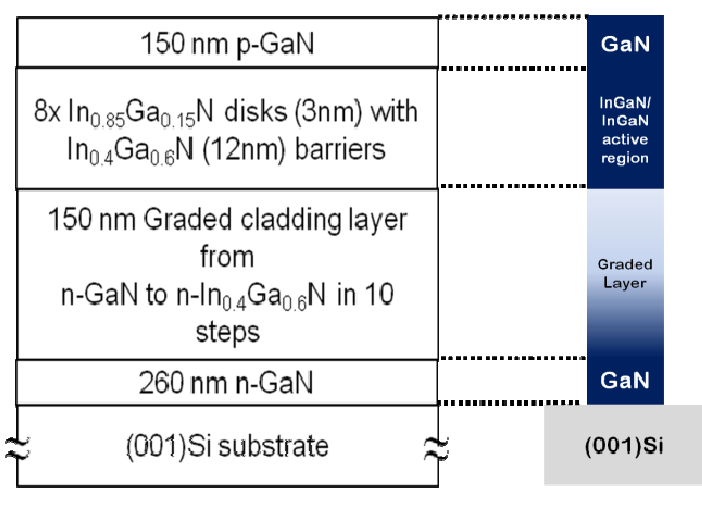

Figure 1: Full laser heterostructure incorporating (a) $\operatorname{In}_{0.85} \mathrm{Ga}_{0.15} \mathrm{~N}$ disks-innanowires emitting at near-infra red. Inset shows schematic of one nanowire. 
which is the active region. After that a $150 \mathrm{~nm} \mathrm{Mg-doped} \mathrm{p-type} \mathrm{GaN} \mathrm{layer} \mathrm{was} \mathrm{grown.} \mathrm{A}$ substrate temperature of $819^{\circ} \mathrm{C}$ was used to grow the n-doped $\mathrm{GaN}$ region under nitrogen rich conditions with a Ga flux of $1.17 \times 10^{-7}$ Torr. The substrate temperature was then varied from $819^{\circ} \mathrm{C}$ to $631^{\circ} \mathrm{C}$ to grow the n-doped graded layer. Throughout the graded layer the Ga and In fluxes were fixed at $1.1 \times 10^{-7}$ Torr and at $4.03 \times 10^{-8}$ Torr, respectively. The substrate temperature was lowered to $442^{\circ} \mathrm{C}$ for the growth of the InGaN disks. The disks were grown at with In flux of $6.04 \times 10^{-8}$ Torr and Ga flux of $2 \times 10^{-8}$ Torr. The substrate temperature was kept constant at $442^{\circ} \mathrm{C}$ for the InGaN barriers, but the In and Ga fluxes were changed to of $3.01 \times 10^{-8}$ Torr and $1.1 \times 10^{-7}$ Torr, respectively. Then the substrate temperature was increased to $800^{\circ} \mathrm{C}$ for the growth of the $\mathrm{p}-\mathrm{GaN}$ region. The $\mathrm{p}-\mathrm{GaN}$ was grown with a Ga flux of $9.63 \times 10^{-8}$ Torr. A higher $\mathrm{Mg}$ flux was used to increase the p-doping level at the final 20nm of p-GaN layer.

A combination of reactive ion etching, photolithography and planarization with parylene has been used to fabricate edge-emitting broad-area nanowire lasers. Parylene also acts as a passivation material to the nanowire and disk regions which increases the relative IQE by $\sim 10-12 \%$ [34]. The laser width was defined to be $50 \mu \mathrm{m}$ in a ridge

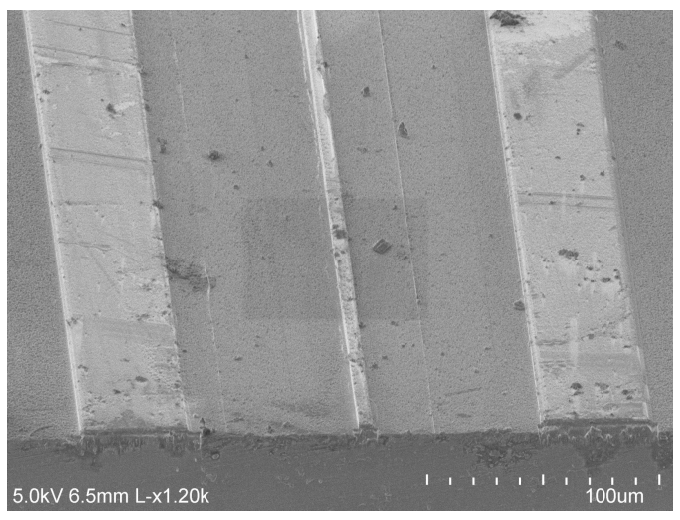
geometry by using two-step mesa etching. The n-contacts were formed using $\mathrm{Al} / \mathrm{Au}$ $(50 \mathrm{~nm} / 200 \mathrm{~nm})$ on the Si substrate surface. Such a device is shown in Fig. 2.

Lasers of lengths varying from 0.5 to $2 \mathrm{~mm}$ were defined by dicing. This was followed by focused ion beam (FIB) etching and the deposition of 2 pairs of

Figure 2: SEM image of the fabricated nanowire lasers with $\operatorname{In}_{0.85} \mathrm{Ga}_{0.15} \mathrm{~N}$ as the disk material. $\mathrm{MgF}_{2} / \mathrm{ZnSe}(235 \mathrm{~nm} / 130 \mathrm{~nm})$ distributed Bragg reflectors (DBRs) to achieve a facet reflectivity of 0.68 at both ends. Measurements were made on $50 \mu \mathrm{m} \times 1 \mathrm{~mm}$ lasers to determine the lightcurrent-voltage (L-I-V) characteristics using pulsed (5\% duty cycle) bias conditions to avoid device heating. No specialized heat sinking or device mounting techniques were used. Figure 3(a)
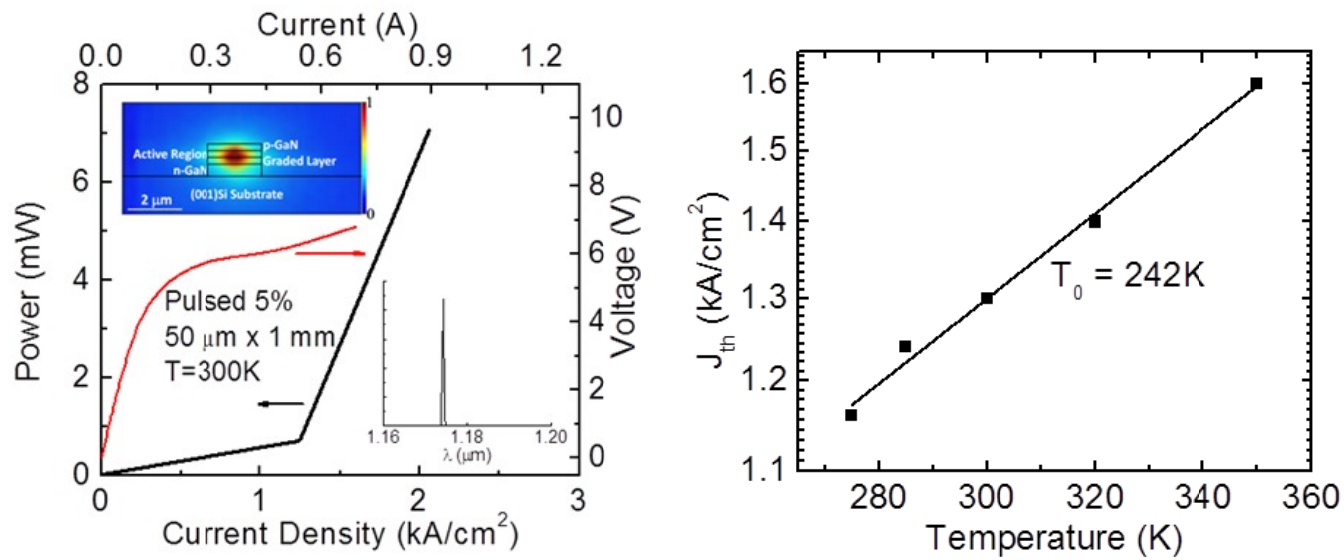

Figure 3: (a) Light-current characteristics of a broad area laser. Top inset shows a near-field image of the laser mode superimposed with the laser heterostructure schematic, and the bottom inset shows electroluminescence spectrum above threshold. (b) Temperature dependence of the threshold current density. 
shows the output characteristics measured at room temperature below and above threshold. A fill factor of 0.85 of the nanowire ensemble and the nanowire area density were used to measure the threshold current density $\left(\mathrm{J}_{\mathrm{th}}\right)$, which was found to be $1.24 \mathrm{kA} / \mathrm{cm}^{2}$. A differential efficiency $\eta_{\mathrm{d}}=$ $0.13 \mathrm{~W} / \mathrm{A}(12.8 \%)$ and a wall-plug efficiency of $0.07 \%$ were also derived from the L-I-V data.

The variation of the threshold current density with temperature was also measured to determine the temperature stability of these lasers. These measurements were done under pulsed bias (5\% duty cycle) conditions with the help of a Peltier cooler. The measured variation is shown in Fig. 3(b). The data were analyzed with the relation: $J_{\text {th }}(T)=J_{\text {tho }} \cdot \exp \left(T / T_{0}\right)$ which results in a $\mathrm{T}_{0}$ value of $242 \mathrm{~K}$. This value of $\mathrm{T}_{0}$ is significantly higher than those of $1.0 \mu \mathrm{m}$ quantum well GaAs-based lasers and is comparable to values of $\mathrm{T}_{0}$ of visible III-nitride nanowire lasers [32], [35]. This clearly indicates that the nanowire lasers are very promising in terms of temperature stability. It is likely that the high value of $\mathrm{T}_{0}$ is a result of the large band offsets in the quantum disk heterostructure, which reduces carrier leakage.

\section{1.3 $\mu \mathrm{M}$ NANOWIRE DIODE LASERS AND DETECTORS}

Figure 4(a) shows a graded refractive index separate confinement heterostructure (GRIN$\mathrm{SCH})$ for nanowire arrays grown on (001)Si substrates for $1.3 \mu \mathrm{m}$ lasers. The nanowire heterostructure is very similar to that for the $1.2 \mu \mathrm{m} \quad$ laser. Graded

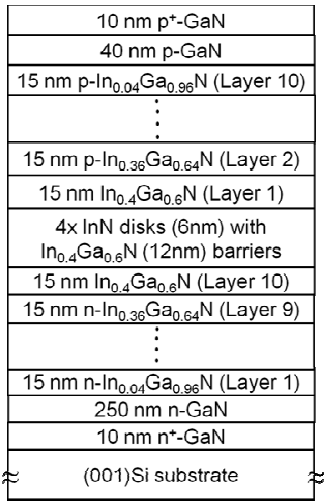

(a)

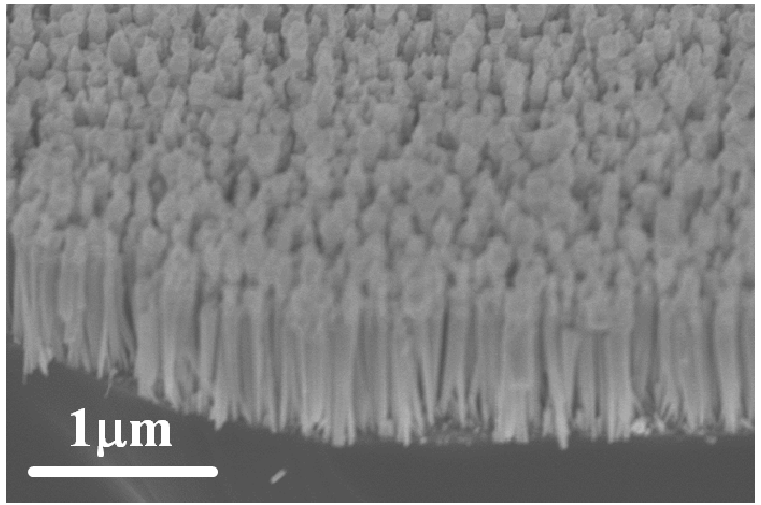

(b)

$\mathrm{In}_{\mathrm{x}} \mathrm{Ga}_{1-\mathrm{x}} \mathrm{N}$ cladding layers

Figure 4: (a) InN/InGaN/GaN nanowire laser heterostructure, (b) SEM image of nanowire heterostructure array.

$(0 \leq x \leq 0.4)$ were placed on both sides of the active region to improve the strain characteristics and the optical confinement. The gain region consists of $4 \mathrm{InN}$ disks of thickness $6 \mathrm{~nm}$ surrounded by $12 \mathrm{~nm} \mathrm{In}_{0.4} \mathrm{Ga}_{0.6} \mathrm{~N}$ barriers, which was grown at $489^{\circ} \mathrm{C}$ and the surrounding graded region was grown at substrate temperatures varying from $631^{\circ} \mathrm{C}\left(\operatorname{In}_{0.4} \mathrm{Ga}_{0.6} \mathrm{~N}\right)$ to $819^{\circ} \mathrm{C}(\mathrm{GaN})$. The heterostructure was designed without an electron blocking layer (EBL). From earlier fabrication it was observed that the electrical characteristics of the fabricated laser diodes having $15 \mathrm{~nm}$ $\mathrm{Al}_{0.15} \mathrm{Ga}_{0.85} \mathrm{~N}$ EBL were substantially inferior to those without the EBL. From scanning electron microscope (SEM) images the height, diameter and density of the nanowires are estimated to be $\sim 700 \mathrm{~nm}, \sim 60 \mathrm{~nm}$, and $\sim 3.2 \times 10^{10} \mathrm{~cm}^{-2}$. Such a SEM image is shown in Fig. 4(b). From these values the fill factor is estimated to be 0.91 for the nanowires.

Low temperature (13K) and room temperature (300K) photoluminescence (PL) measurements were made on the half laser heterostructure. In these half laser heterostructures growth was terminated after four periods of InN disks and $\mathrm{In}_{0.4} \mathrm{Ga}_{0.6} \mathrm{~N}$ barriers and grown with the optimized parameters described earlier. The spectra recorded at $13 \mathrm{~K}$ and $300 \mathrm{~K}$ are illustrated in Fig. 5. An approximate value of the radiative efficiency $\left(\eta_{\mathrm{r}}\right)$ of $67 \%$ is estimated from the PL 
peak intensities at $300 \mathrm{~K}$ and $13 \mathrm{~K}$, assuming that non-radiative recombination channels are frozen at the latter temperature [36].

Steady state measurements were made on the discrete nanowire lasers mounted on a copper block without any additional heat sinking. The laser temperature was maintained at the ambient temperature $(\sim 300 \mathrm{~K})$ with active biasing using a Peltier cooler. Figure 9 shows the light-current (L-I) characteristics of a broad area $(50 \mu \mathrm{m} \times 1.5 \mathrm{~mm})$ laser measured in both continuous wave (cw) and pulsed bias mode $(20 \mu \mathrm{s}$ pulses, $1 \%$ duty cycle). The slope efficiency is slightly higher for the pulsed biasing mode compared to the $\mathrm{cw}$

mode and found to be $0.12 \mathrm{~W} / \mathrm{A}$. Spectral measurements of the laser output were also performed and the output at a bias of $\mathrm{I}_{\text {inj }}=1.2 \mathrm{I}_{\text {th }}$ is shown in the inset of Fig. 6(a). The peak emission wavelength is at $1.33 \mu \mathrm{m}$. There is

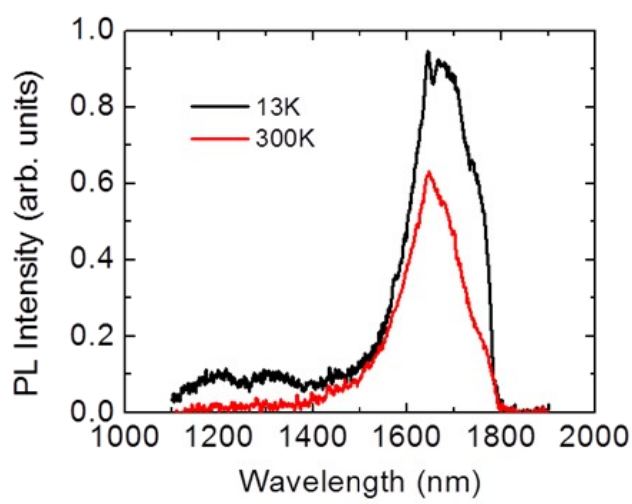

Figure 5: Measured room temperature and low temperature photoluminescence spectra obtained from a nanowire array sample. Growth was terminated after the InN disk based active region for this sample.

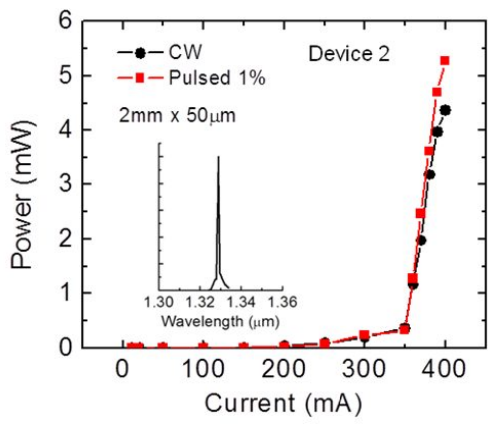

(a)

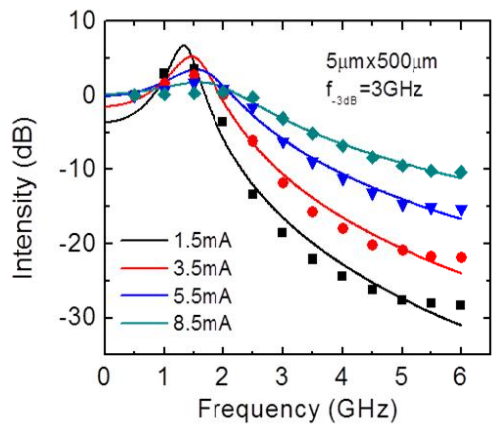

(b)

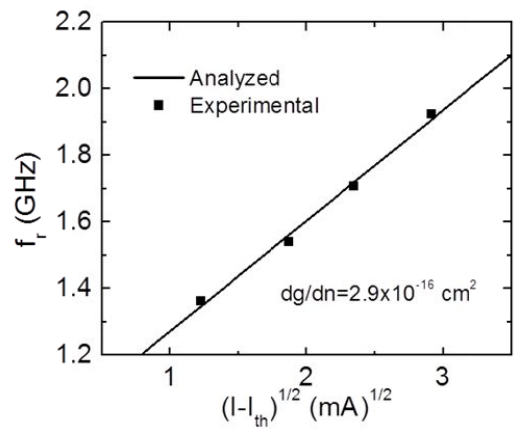

(c)

Figure 6: (a) Measured output light-current (L-I) characteristics of edge-emitting InN/InGaN/GaN dot-innanowire laser diode. Inset shows the electroluminescence spectrum above threshold, (b) High frequency response of an edge-emitting $1.3 \mu \mathrm{m}$ nanowire laser at different bias currents. The values of currents shown are above the threshold current. (c) Resonance frequency variation as a function of square root of injection current as determined from $b$.

clearly a strong blue shift of this EL emission compared to the PL emission peak which was measured at $\sim 1.6 \mu \mathrm{m}$. This is believed to be due to the injection-related quantum confined Stark effect.

Small-signal modulation measurements have been performed on discrete $5 \mu \mathrm{m} \times 500 \mu \mathrm{m}$ ridge waveguide lasers. The modulation response was measured with a Hewlett-Packard 8350B sweep oscillator, Hewlett-Packard 83599A RF plug-in, bias T, low-noise amplifier, Newport high speed detector and Hewlett-Packard 8593A spectrum analyzer. Figure 6(b) shows the frequency response for varying $\mathrm{CW}$ injection currents $\left(\mathrm{I}-\mathrm{I}_{\mathrm{th}}\right)$. Each point represents the peak value of the spectral output at the corresponding frequency. The solid curves represent calculated modulation response in accordance with a response function incorporating damping limited operation. The data have been analyzed with the response function, 


$$
|R(f)|^{2} \propto \frac{1}{\left(f^{2}-f_{r}^{2}\right)+\left(\frac{\gamma_{d}}{2 \pi}\right)^{2} f^{2}}
$$

where $f_{r}$ is the resonance frequency and $\gamma_{d}$ is the damping factor. From this measurement, a $-3 \mathrm{~dB}$ modulation bandwidth of $3.0 \mathrm{GHz}$ has been derived for an injection current of $\left(\mathrm{I}-\mathrm{I}_{\mathrm{th}}\right)=8.5 \mathrm{~mA}$. Figure 6(c) shows a plot of $\mathrm{f}_{\mathrm{r}}$ versus $\left(\mathrm{I}-\mathrm{I}_{\mathrm{th}}\right)^{1 / 2}$, from which a slope of $0.33 \mathrm{GHz} /(\mathrm{mA})^{1 / 2}$ is derived. This value of slope is used to calculate the differential gain $\mathrm{dg} / \mathrm{dn}$ in accordance with the relation,

$$
f_{r}=\frac{1}{2 \pi}\left[\frac{v_{g} \Gamma\left(I-I_{t h}\right) \frac{d g}{d n} \eta_{r}}{V_{a c t} q}\right]^{\frac{1}{2}}
$$

Here $\Gamma$ is the mode confinement factor, $\eta_{\mathrm{r}}$ is the radiative efficiency, $\mathrm{v}_{\mathrm{g}}$ is the photon group velocity and $V_{\text {act }}$ is the active volume of the gain medium. Transfer-matrix-element method based simulations have been performed to determine the value of $\Gamma$. It has been found that $\Gamma=$ 0.0137. The simulation assumes the laser as a planar device, which is evident from plane wave propagation based simulations described earlier. The value of $\eta_{\mathrm{r}}$ is taken to be $67 \%$ as determined from the PL measurements. The value of $\mathrm{dg} / \mathrm{dn}$ is then calculated to be $2.9 \times 10^{-16}$ $\mathrm{cm}^{2}$.

\section{MONOLITHIC PHOTONIC INTEGRATED CIRCUIT}

Discrete guided wave dot-in-nanowire photodiodes were also measured in order to determine their performance characteristics. To measure the photocurrent spectra of these photodiodes, they were reverse biased and end-fired with light from a broadband source dispersed by a $0.125 \mathrm{~m}$ CM110 monochromator with a spectral resolution of $0.2 \mathrm{~nm}$. The spectral measurements were performed at room temperature. The value of the reverse bias applied to the photodiode was varied to understand the evolution of the photocurrent

spectra with bias. The value of the photocurrent was measured with a Keithley 6487 picoammeter having a

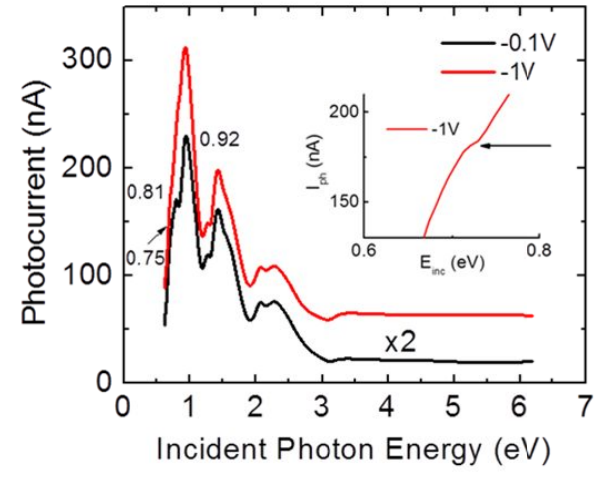

Figure 7: Photocurrent spectra of nanowire based photodetectors. resolution of $1 \mathrm{pA}$. Figure 7 shows the measured spectra. Several shoulders and peaks can be observed at energies close to the absorption edge.

The fabrication of the photonic integrated circuit follows near-identical steps. This has enabled us to fabricate both the laser and the detector on the same chip thus forming the photonic integrated circuit. The dielectric waveguide in between the nanowire laser and detector is formed by selective etching of the nanowires and deposition of $400 \mathrm{~nm} \mathrm{SiO}{ }_{2}$ followed by $400 \mathrm{~nm}$ of $\mathrm{Si}_{3} \mathrm{~N}_{4}$. The multilayer waveguide has relatively small waveguide loss. For the laser, the facet away from the waveguide was made reflective by FIB etching and subsequent deposition of $\mathrm{MgF}_{2} / \mathrm{ZnSe} \mathrm{DBR}$ layers and the facet coupled to the waveguide was made reflective with 4 pairs of air/nanowire-paralyne DBR layers, also formed by FIB etching (shown in the inset of Fig. $8(\mathrm{a}))$. For the detector, $\sim 220 \mathrm{~nm}$ of anti-reflective $\mathrm{SiO}_{2}$ was deposited on the facet not coupled to 
the waveguide. The coupling grooves between the waveguide and the two active devices are $\sim 5 \mu \mathrm{m}$. The complete photonic circuit with sections of the laser and detector along with the waveguide can be seen in Fig. 8(a).

The
variation of
the detector
photocurrent
along with the
laser injection
current can be
seen in Fig.
8(b). For this
measurement,

the laser has been excited with a dc pulsed (20 $\mu$ s pulses, $1 \%$ duty cycle)

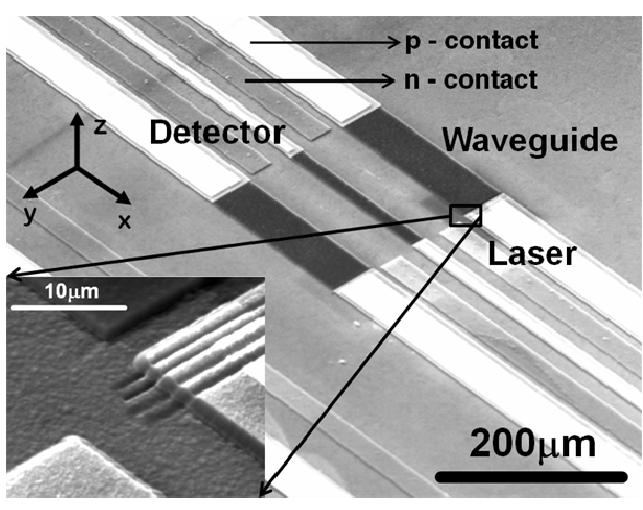

(a)

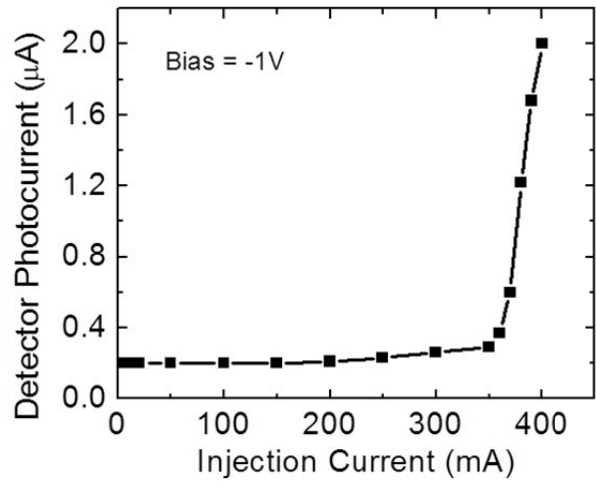

(b)

Figure 8: (a) SEM image of III-nitride nanowire array photonic integrated circuit fabricated directly on (001)Si showing all the major components of the circuit. A magnified image of the air/nanowire DBR mirror is shown in the inset. (b) Variation of photocurrent of the photodiode as a function of laser injection current. Both optoelectronic devices are parts of the same photonic integrated circuit.

injection bias. The photocurrent response of the detector to the light emitted by the laser and coupled to the waveguide between the two devices has been recorded. The detector was kept at a reverse bias of $-1 \mathrm{~V}$. For this measurement, both the laser and detector used have a width of 50 $\mu \mathrm{m}$, and they have a length of $2 \mathrm{~mm}$ and $1 \mathrm{~mm}$, respectively. The $\mathrm{SiO}_{2} / \mathrm{Si}_{3} \mathrm{~N}_{4}$ waveguide in between is $200 \mu \mathrm{m}$ long and $50 \mu \mathrm{m}$ wide. The photodiode reverse saturation current follows the laser light-current characteristics quite well. A 10 -fold increase $(0.2 \mu \mathrm{A}$ to $2 \mu \mathrm{A})$ in the reverse saturation current of the photodiode has been measured. It is expected that the light coupled into the photodiode can be substantially increased by improved design and fabrication. Better FIB techniques and facet coating can also improve the amount of light coupling.

\section{CONCLUSION}

GaN-based InGaN/GaN disk-in-nanowire arrays grown on silicon substrates have properties which can be exploited to realize optoelectronic devices for silicon photonics and other applications. We have described here the epitaxial growth, fabrication and properties of discrete nanowire array diode lasers and detectors and a monolithically integrated photonic integrated circuit in which the two active devices are integrated with a passive dielectric waveguide.

\section{ACKNOWLEDGMENT}

This work was supported by the National Science Foundation under Grants ECCS1648870 and DMR-1120923 (MRSEC program). 


\section{References}

[1] A. Kikuchi, M. Kawai, M. Tada, and K. Kishino, "InGaN/GaN Multiple Quantum Disk Nanocolumn Light-Emitting Diodes Grown on (111) Si Substrate," Jpn. J. Appl. Phys., vol. 43, no. 12A, p. L1524, Nov. 2004.

[2] W. Guo, A. Banerjee, P. Bhattacharya, and B. S. Ooi, "InGaN/GaN disk-in-nanowire white light emitting diodes on (001) silicon," Appl. Phys. Lett., vol. 98, no. 19, p. 193102, May 2011.

[3] H. P. T. Nguyen, K. Cui, S. Zhang, S. Fathololoumi, and Z. Mi, "Full-color InGaN/GaN dot-in-a-wire light emitting diodes on silicon," Nanotechnology, vol. 22, no. 445202, pp. 15, Oct. 2011.

[4] X. Duan and C. M. Lieber, "Laser-Assisted Catalytic Growth of Single Crystal GaN Nanowires," J. Am. Chem. Soc., vol. 122, no. 1, pp. 188-189, Jan. 2000.

[5] L. Cerutti, J. Ristić, S. Fernández-Garrido, E. Calleja, A. Trampert, K. H. Ploog, S. Lazic, and J. M. Calleja, "Wurtzite GaN nanocolumns grown on $\mathrm{Si}(001)$ by molecular beam epitaxy," Appl. Phys. Lett., vol. 88, no. 21, p. 213114, May 2006.

[6] T. Kuykendall, P. Ulrich, S. Aloni, and P. Yang, "Complete composition tunability of InGaN nanowires using a combinatorial approach," Nat. Mater., vol. 6, no. 12, pp. 951956, Dec. 2007.

[7] S. Fernández-Garrido, J. Grandal, E. Calleja, M. A. Sánchez-García, and D. López-Romero, "A growth diagram for plasma-assisted molecular beam epitaxy of GaN nanocolumns on Si(111)," J. Appl. Phys., vol. 106, no. 12, p. 126102, Dec. 2009.

[8] A. Armstrong, Q. Li, K. H. A. Bogart, Y. Lin, G. T. Wang, and A. A. Talin, "Deep level optical spectroscopy of GaN nanorods," J. Appl. Phys., vol. 106, no. 5, p. 053712, Sep. 2009.

[9] C. Cheze, L. Geelhaar, O. Brandt, W. M. Weber, H. Riechert, S. Munch, R. Rothemund, S. Reitzenstein, A. Forchel, T. Kehagias, P. Komninou, G. P. Dimitrakopulos, and T. Karakostas, "Direct Comparison of Catalyst-Free and Catalyst-Induced GaN Nanowires," Nano Res., vol. 3, no. 7, pp. 528-536, Jul. 2010.

[10] F. Glas, "Critical dimensions for the plastic relaxation of strained axial heterostructures in free-standing nanowires," Phys. Rev. B, vol. 74, no. 12, p. 121302, Sep. 2006.

[11] S. D. Hersee, A. K. Rishinaramangalam, M. N. Fairchild, L. Zhang, and P. Varangis, "Threading defect elimination in GaN nanowires," J. Mater. Res., vol. 26, no. 17, pp. 22932298, Sep. 2011.

[12] W. Guo, M. Zhang, P. Bhattacharya, and J. Heo, "Auger Recombination in III-Nitride Nanowires and Its Effect on Nanowire Light-Emitting Diode Characteristics," Nano Lett., vol. 11, no. 4, pp. 1434-1438, Apr. 2011.

[13] C.-F. Huang, C.-F. Lu, T.-Y. Tang, J.-J. Huang, and C. C. Yang, "Phosphor-free white-light light-emitting diode of weakly carrier-density-dependent spectrum with prestrained growth of InGaNGaN quantum wells," Appl. Phys. Lett., vol. 90, no. 15, p. 151122, Apr. 2007. 
[14] Y.-H. Cho, G. H. Gainer, A. J. Fischer, J. J. Song, S. Keller, U. K. Mishra, and S. P. DenBaars, "“S-shaped"" temperature-dependent emission shift and carrier dynamics in InGaN/GaN multiple quantum wells," Appl. Phys. Lett., vol. 73, no. 10, pp. 1370-1372, Sep. 1998.

[15] F. B. Naranjo, M. A. Sánchez-García, F. Calle, E. Calleja, B. Jenichen, and K. H. Ploog, "Strong localization in InGaN layers with high In content grown by molecular-beam epitaxy," Appl. Phys. Lett., vol. 80, no. 2, pp. 231-233, Jan. 2002.

[16] C. Kölper, M. Sabathil, M. Mandl, M. Strassburg, and B. Witzigmann, "All-InGaN Phosphorless White Light Emitting Diodes: An Efficiency Estimation,” J. Light. Technol., vol. 30, no. 17, pp. 2853-2862, Sep. 2012.

[17] W. Guo, M. Zhang, A. Banerjee, and P. Bhattacharya, "Catalyst-Free InGaN/GaN Nanowire Light Emitting Diodes Grown on (001) Silicon by Molecular Beam Epitaxy," Nano Lett., vol. 10, no. 9, pp. 3355-3359, Sep. 2010.

[18] A. A. Setlur, "Phosphors for LED-based Solid-State Lighting," Electrochem. Soc. Interface, vol. 18, no. 4, pp. 32-36, 2009.

[19] Y. Narukawa, I. Niki, K. Izuno, M. Yamada, Y. Murazaki, and T. Mukai, "PhosphorConversion White Light Emitting Diode Using InGaN Near-Ultraviolet Chip," Jpn. J. Appl. Phys., vol. 41, no. 4A, p. L371, Apr. 2002.

[20] J. K. Park, C. H. Kim, S. H. Park, H. D. Park, and S. Y. Choi, "Application of strontium silicate yellow phosphor for white light-emitting diodes," Appl. Phys. Lett., vol. 84, no. 10, pp. 1647-1649, Mar. 2004.

[21] S. C. Allen and A. J. Steckl, "A nearly ideal phosphor-converted white light-emitting diode," Appl. Phys. Lett., vol. 92, no. 14, p. 143309, Apr. 2008.

[22] S. Ye, F. Xiao, Y. X. Pan, Y. Y. Ma, and Q. Y. Zhang, "Phosphors in phosphor-converted white light-emitting diodes: Recent advances in materials, techniques and properties," Mater. Sci. Eng. R Rep., vol. 71, no. 1, pp. 1-34, Dec. 2010.

[23] D. A. Steigerwald, J. C. Bhat, D. Collins, R. M. Fletcher, M. O. Holcomb, M. J. Ludowise, P. S. Martin, and S. L. Rudaz, "Illumination with solid state lighting technology," IEEE J. Sel. Top. Quantum Electron., vol. 8, no. 2, pp. 310-320, Mar. 2002.

[24] J. Baur, F. Baumann, M. Peter, K. Engl, U. Zehnder, J. Off, V. Kuemmler, M. Kirsch, J. Strauss, R. Wirth, K. Streubel, and B. Hahn, "Status of high efficiency and high power ThinGaN®-LED development,” Phys. Status Solidi C, vol. 6, no. S2, pp. S905-S908, Jun. 2009.

[25] I. H. Cho, G. Anoop, D. W. Suh, S. J. Lee, and J. S. Yoo, "On the stability and reliability of $\mathrm{Sr}_{1-\mathrm{x}} \mathrm{Ba}_{\mathrm{x}} \mathrm{Si}_{2} \mathrm{O}_{2} \mathrm{~N}_{2}$ :Eu ${ }^{2+}$ phosphors for white LED applications," Opt. Mater. Express, vol. 2, no. 9, pp. 1292-1305, Sep. 2012.

[26] S. Nakamura, M. Senoh, N. Iwasa, and S. Nagahama, "High-Brightness InGaN Blue, Green and Yellow Light-Emitting Diodes with Quantum Well Structures," Jpn. J. Appl. Phys., vol. 34, no. 2, pp. L797-L799, May 1995.

[27] K. Kishino, A. Kikuchi, H. Sekiguchi, and S. Ishizawa, "InGaN/GaN nanocolumn LEDs emitting from blue to red," Proc. SPIE, vol. 6473, p. 64730T, Feb. 2007.

[28] J. B. Schlager, K. A. Bertness, P. T. Blanchard, L. H. Robins, A. Roshko, and N. A. Sanford, "Steady-state and time-resolved photoluminescence from relaxed and strained GaN nanowires grown by catalyst-free molecular-beam epitaxy," J. Appl. Phys., vol. 103, no. 12, p. 124309, Jun. 2008. 
[29] R. Calarco, M. Marso, T. Richter, A. I. Aykanat, R. Meijers, A. v.d. Hart, T. Stoica, and H. Lüth, "Size-dependent Photoconductivity in MBE-Grown GaN-Nanowires," Nano Lett., vol. 5, no. 5, pp. 981-984, May 2005.

[30] H. P. T. Nguyen, M. Djavid, K. Cui, and Z. Mi, "Temperature-dependent nonradiative recombination processes in GaN-based nanowire white-light-emitting diodes on silicon," Nanotechnology, vol. 23, no. 19, p. 194012, 2012.

[31] S. Jahangir, T. Schimpke, M. Strassburg, K. A. Grossklaus, J. M. Millunchick, and P. Bhattacharya, "Red-emitting $(\lambda=610 \mathrm{~nm}) \mathrm{In}_{0.51} \mathrm{Ga}_{0.49} \mathrm{~N} / \mathrm{GaN}$ disk-in-nanowire light emitting diodes on silicon," IEEE J. Quantum Electron., vol. 50, no. 7, pp. 530-537, Jul. 2014.

[32] T. Frost, S. Jahangir, E. Stark, S. Deshpande, A. Hazari, C. Zhao, B. S. Ooi, and P. Bhattacharya, "Monolithic electrically injected nanowire array edge-emitting laser on (001) silicon," Nano Lett., vol. 14, no. 8, pp. 4535-4541, Jul. 2014.

[33] S. Jahangir, I. Pietzonka, M. Strassburg, and P. Bhattacharya, "Monolithic phosphor-free InGaN/GaN quantum dot wavelength converter white light emitting diodes," Appl. Phys. Lett., vol. 105, no. 11, p. 111117, Sep. 2014.

[34] S. Jahangir, M. Mandl, M. Strassburg, and P. Bhattacharya, "Molecular beam epitaxial growth and optical properties of red-emitting $(\lambda=650 \mathrm{~nm}) \mathrm{InGaN} / \mathrm{GaN}$ disks-in-nanowires on silicon," Appl. Phys. Lett., vol. 102, no. 7, p. 071101, Feb. 2013.

[35] S. Jahangir, T. Frost, A. Hazari, L. Yan, E. Stark, T. LaMountain, J. Mirecki-Millunchick, B. S. Ooi, and P. Bhattacharya, "Small signal modulation characteristics of red-emitting $(\lambda$ $=610 \mathrm{~nm}$ ) III-nitride nanowire array lasers on (001) silicon," Appl. Phys. Lett., vol. 106, no. 071108 , Feb. 2015.

[36] Ž. Gačević, A. Das, J. Teubert, Y. Kotsar, P. K. Kandaswamy, T. Kehagias, T. Koukoula, P. Komninou, and E. Monroy, "Internal quantum efficiency of III-nitride quantum dot superlattices grown by plasma-assisted molecular-beam epitaxy," J. Appl. Phys., vol. 109, no. 10, p. 103501, May 2011. 